\title{
IS STELLAR WIND MASS LOSS DURING CORE HYDROGEN BURNING IMPORTANT FOR EVOLUTION.
}

\author{
R. BLOMME $^{(1)}$, D. VANBEVEREN ${ }^{(2)}$, W. VAN RENSBERGEN ${ }^{(2)}$. \\ (1): Koninklijke Sterrenwacht, Brussels, Belgium, \\ (2): Dept. of Physics, VUB, Brussels, Belgium.
}

SUMMARY. The influence of wind mass loss during core hydrogen burning on the evolution of massive stars can be investigated using a relation between $\dot{M}-\mathrm{L}-\mathrm{M}-\mathrm{T}_{\text {eff }}$ based on

a. observed $\left(\dot{\mathrm{M}}, \mathrm{L}, \mathrm{T}_{\text {eff }}\right)$ values and masses which are determined from evolutionary computations (e.g. Nieuwenhuijzen and de Jager, 1990, A.\&A. 231, 134),

b. the theory of radiation driven stellar wind. With the cooking recipe of Kudritzki et al.(1989, A.\&A. 219, 205), we determined the predicted $M$ of a star at the beginning, in the middle and at the end of core hydrogen burning using the $20 \mathrm{M}_{\odot}, 40 \mathrm{M}_{\odot}, 60 \mathrm{M}_{\odot}$ and $85 \mathrm{M}_{\odot}$ evolutionary computations of Maeder and Meynet $(1987, A . \& A .182,243)$. The following two formulae then give $\dot{M}$ as a function of $L-T_{\text {eff }}$ and $M$ with a sigma value lower than 0.04 , i.e.

$$
\begin{gathered}
\log (-\dot{\mathrm{M}})=-13.18+1.88 \log \mathrm{L}-\log \mathrm{M}-0.4 \log \mathrm{T}_{\text {eff }} \\
\log (-\dot{\mathrm{M}})=-10.31+1.51 \log \mathrm{L}-0.2 \log \mathrm{M}-0.88 \log \mathrm{T}_{\mathrm{eff}} \\
(\mathrm{M}, \mathrm{L} \text { and } \mathrm{M} \text { are in solar units })
\end{gathered}
$$

Formula (1) is determined by assigning equal weights to all points. Formula (2) however gives the relation between $\mathrm{M}$ and the stellar parameters when the different points are weighted according to the function $\mathrm{M}_{\mathrm{i}}{ }^{-3.5}\left(\mathrm{M}_{\mathrm{i}}\right.$ stands for the ZAMS mass of the corresponding evolutionary track); the function describes the observed number density of massive stars corrected for observational selection (Humphreys and McElroy, 1984, Ap. $J .284,565$ ). With these formulae, evolution with convective core overshooting according to the model of Maeder and Meynet $(1987, A . \& A .182,243)$ predicts that a $40 \mathrm{M}_{\Theta}\left(60 \mathrm{M}_{\odot}, 85 \mathrm{M}_{\Theta}\right)$ star will loose less than $2.7 \mathrm{M}_{\odot}\left(5.2 \mathrm{M}_{\Theta}, 7.6 \mathrm{M}_{\odot}\right.$ respectively) during core hydrogen burning, i.e.

if the theory of radiation driven stellar wind in its present form accurately predicts the mass loss of a massive core hydrogen burning star, then one can conclude that it is a fairly unimportant process as far as evolution is concerned.

The results summarised here will be published in Astron. Astrophys.

K. A. van der Hucht and B. Hidayat (eds.),

Wolf-Rayet Stars and Interrelations with Other Massive Stars in Galaxies, 547.

(C) 1991 IAU. Printed in the Netherlands. 